\title{
Stereopsis and subjective contours
}

\author{
M. B. SIMMONDS \\ University of Canterbury, Christchurch, New Zealand
}

\begin{abstract}
Ten Ss rated perceived depth and contour clarity of figures containing binocularly disparate subjective contours. There was no tendency for stereoscopic depth cues to enhance the perceived clarity of subjective contours. Disparity cues that were incompatible with monocular depth cues reduced the depth sensation but did not affect contour clarity. Although subjective contours can be perceived stereoscopically, they are seen in less depth than real contours with the same degree of horizontal disparity.
\end{abstract}

In an analysis of the conditions that give rise to the perception of subjective contours in the absence of abrupt brightness gradients, Coren (1972) has discussed the significance of both form disparity and monocular depth cues. He presents as evidence for his explanation of the phenomenon a series of illustrations and demonstrations which are consistent with the view that subjective contours appear when a two-dimensional display is interpreted by the $O$ to contain surfaces or planes that are in depth relative to each other. Referring to illustrations that are similar to Fig. 1 below, Coren concludes that subjective contours appear when the $\mathrm{O}$ is able visually to reorganize a chaotic collection of complex two-dimensional elements into a simple and easily coded three-dimensional array of meaningful or symmetrical elements. He explores in particular detail the effect of two types of depth cue-shading and interposition. A major assumption made by Coren is that subjective contours arise because the area bounded by the contour is seen to lie in a different plane of depth from other parts of the figure; however, he offers little empirical evidence to support this claim. Furthermore, the effect of what is perhaps the most potent depth cue of all, horizontal disparity in binocularly presented images, does not appear to have been considered.

Our observations suggest that there are two stages involved in the perception of subjective contours. The first step involves an active exploratory stage of organizing the display into an orderly, symmetrical, and meaningful pattern; this stage has been discussed in detail by Coren.

The unique feature of figures that are known to generate subjective contours is that at least one of the possible structurings of the display is dependent upon the addition of contours to complete the organization of planes or areas within the figures. Once the potential for organization into a simple figure has been grasped by the $O$, he enters a second stage, which involves the continued perception of a stable, orderly display. However, in order to maintain this percept, it is essential for him to add the necessary contours to his percept. It is this process of actively adding cues that are not present in the display, but which are nevertheless essential for its maintenance as a stable percept, which leads him to "see" a contour line bridging the uncompleted sections of the stimulus. It is as if the $O$ holds an image of the figure in his head, which he then projects out onto the physical object that he is observing.

It is generally accepted that interchanging the left and right fields of a stereogram results in the production of disparity cues which are compatible with reversal in perceived depth when the stereogram is viewed binocularly. However, the expected depth reversal does not generally occur if other depth cues in the display (such as interposition and shading) remain incompatible with the perception of depth reversal. This interaction between the stereoscopic and monocular depth cues appears to have been first reported by Schriever (1925) and has been more recently discussed by Gregory (1970). If it can be shown empirically that interchanging left and right images of a stereoscopic pair does not reverse the perceived depth relationships, then this would support the hypothesis that there are monocular depth cues in the display that are concordant with a possible depth interpretation, and that, furthermore, these monocular depth cues are sufficiently powerful to mask or compensate for the depth cues given by the disparity in the reversed pair of images.

Figure 1 is a stereoscopic version of the type of figure that has been created by Kanizsa (1955) and discussed by Coren. When it is viewed stereoscopically, the central white square appears to float forward of the background, the square itself being defined (as in the situation where the figure is viewed monocularly) by subjective contours. These subjective contours are similar to the anomalous contours described by Lawson and Gulick (1967) and by Shipley (1965); however, in this case, the contours can be seen under monocular as well as under stereoscopic viewing conditions.

\section{METHOD}

Twenty-two stimuli were prepared by systematically altering the relationships between the parts shown in Fig. 1 to produce the following variations. Series 1 consisted of a set of 11 pairs in which the contour surrounding the central white square was clearly marked in by lines (Fig. 2). The spatial location of the white square ranged from lying in depth behind the black 

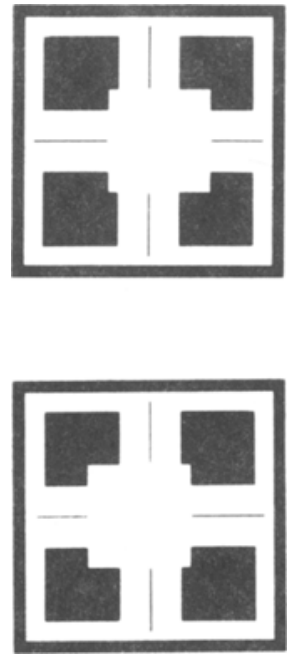

Fig. 1.
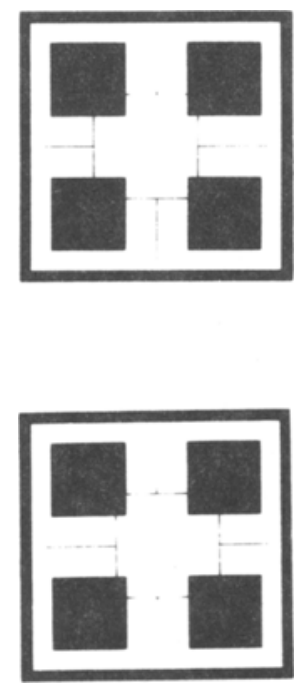

Fig. 2.

squares (as shown in Fig. 2) through a condition in which it lay in the same plane as the black squares, and up to a condition where the white square lay in front of the black squares with the same level of disparity as that shown for the subjective contour in Fig. 1. Series 2 was a set of 10 pairs in which the white square had no contour marked in the regions between the black squares (as in Fig. 1). In addition, a pair was constructed in which there were no interposition cues present in the display (apart from the crossed hair lines), and hence there was zero tendency to perceive a central white square at the location in which the white square appeared in all of the other stimuli (Fig. 3).

An experiment was designed to explore the effect of the addition of disparity cues on the perceived clarity of subjective contours. Coren's explanation of the origin of subjective contours in nonstereoscopic patterns states that monocular depth cues (particularly interposition) in the stimulus display give rise to the perceived contour. If this is so, then it is reasonable to postulate that the perceived clarity of the contour will be a function of the number of appropriate depth cues present in the display. We therefore predict that the addition of disparity cues will enhance the clarity of the perceived contour. In addition, if Coren's monocular patterns yield a display containing apparent depth, then reversal of this perceived depth (by reversing the disparity cues) should attenuate the tendency to perceive a subjective contour.

Two 11-point scales were constructed. Scale 1 was concerned with the clarity or definition of the contour surrounding the central white square in the figures. Its end points were defined by illustrations representing the contour as marked in Fig. 2 at one pole (maximum contour definition) with the opposite pole defined by a diagram representing zero tendency to perceive a white square surrounded by any kind of contour (as in Fig. 3). The midpoint of the scale was indicated by a diagram in which the white square was surrounded by a dotted line. Scale 2 was concerned with the depth relationship between the plane in which the white square lay and the plane of the black squares. Its two poles were defined by perspective drawings representing projection of the white square behind the black squares and projection of the white square forward of the black squares. Its midpoint was defined by a diagram in which the black squares and the white square lay in the same plane.

All pairs of stimuli were presented in a lenticular stereoscope of the type described by Valyus (1966), which was modified to allow left and right fields of the display to be interchanged. The stimuli were printed on high-contrast photographic paper, with the black frame surrounding the diagrams measuring $6.5 \mathrm{~cm}$ in length. The visual angle subtended by the stimuli varied between 12 and $14 \mathrm{deg}$, as a function of the distance between the $O$ 's eye and the stimulus held in the stereoscope. The modal setting subtended an angle of $13 \mathrm{deg}$; individual Os were permitted to select the most comfortable viewing distance before the experiment commenced, but were not permitted to alter the selected setting during the experiment. The luminance of the white portions of the stimulus under the viewing conditions employed was approximately $30 \mathrm{~lm} / \mathrm{m}^{2}$.

Each of 10 volunteer undergraduate students made two judgments of each stimulus on the depth scale and two judgments on the contour-clarity scale; the stimuli were presented cyclically in random order, with 22 trials separating judgment on the depth scale and judgment on the contour-clarity scale for a given stimulus.

The Ss were instructed that they would be required to rate two separate aspects of the stimulus display on two different scales, a depth scale and a contour clarity scale. It was stressed that $S s$ were to base their depth judgments entirely on the perceived difference between the plane in which the white square lay and the plane of the black squares when judging depth. Contour clarity was similarly described as the perceived definition or clarity of the boundary surrounding the central white square. The two extremes in depth, and the two extremes in contour definition (as described and illustrated above) were then presented in the stereoscope; Ss were advised that, in each case, these two extremes were represented by the opposite poles of the appropriate scale, and that all stimuli to be presented would lie between the extremes that had been demonstrated to them.

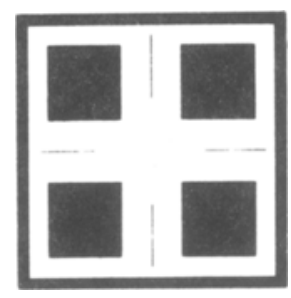

Fig. 3. 


\section{RESULTS}

Data were pooled across Ss and in some cases across stimuli of a given type, and a series of two-sample repeated measures $t$ tests were conducted. The pooled data appear in Tables 1 and 2. Preliminary checks on the reliability of the two scales as a means of indicating perceived differences in depth and contour-clarity revealed that the Ss had little difficulty in translating their percepts into scale values. Comparison of judgments on figures without disparity and judgments on figures with disparity (at two different levels of disparity) for both subjective and marked contours combined indicated that stimuli with disparity were seen in significantly greater depth than were figures with no disparity cue (the mean depth judgments appear in Table 1; $t=5.27, t=5.63$, with $39 \mathrm{df}, \mathrm{p}<.001$ in both cases). Four of the stimuli in the series involved incompatibility between the direction of depth given by the disparity cue and the direction given by the interposition cue. These incompatibilities were obtained by reversing left and right fields of the stereogram. In all cases, there was a significant reduction in depth when stimuli involving incompatible depth cues were compared with the corresponding unreversed stimuli (Table $1 ; t$ ranged between 3.56 and 5.06 , $\mathrm{df}=19$, $\mathrm{p}<.01$ ), again suggesting that the depth scale was used reliably. Likewise, stimuli with contours marked in were given higher overall ratings on the contour clarity scale than figures with subjective contours (Table $1 ; \mathrm{t}=14.55$, $\mathrm{df}=39, \mathrm{p}<.001$ ), and the 10 figures with subjective contours were given significantly higher ratings when each of the 10 was compared in turn with the zero-contour condition shown in Fig. 3. (The mean depth and contour ratings for the pattern shown in Fig. 3 were 0.10 and 0.20 , respectively; $t$ ranged between 6.06 and 8.18 in all cases, with $19 \mathrm{df}$ and $\mathrm{p}<.001$.)

In no case does the disparity cue appear to have

Table 1

Pooled Means and Standard Deviations (in Parentheses) of Judged Depth and Contour Clarity for Both Real and Subjective Contours*

\begin{tabular}{|c|c|c|c|c|c|}
\hline \multicolumn{2}{|c|}{$\begin{array}{l}\text { No Disparity } \\
\text { (Monocular } \\
\text { Cues Only) }\end{array}$} & \multicolumn{2}{|c|}{$\begin{array}{c}\text { Disparity Cue } \\
\text { Incompatible } \\
\text { With Monoc- } \\
\text { ular Cues } \\
\end{array}$} & \multicolumn{2}{|c|}{$\begin{array}{c}\text { Disparity Cue } \\
\text { Compatible } \\
\text { With Monoc- } \\
\text { ular Cues }\end{array}$} \\
\hline Depth & Contour & Depth & Contour & Depth & Contour \\
\hline \multicolumn{6}{|c|}{ Square Bound by Subjective Contours } \\
\hline $\begin{array}{c}1.25 \\
(1.45)\end{array}$ & $\begin{array}{c}4.60 \\
(2.69)\end{array}$ & $\begin{array}{c}0.83 \\
(1.25)\end{array}$ & $\begin{array}{c}4.48 \\
(2.52)\end{array}$ & $\begin{array}{c}2.95 \\
(1.27)\end{array}$ & $\begin{array}{c}5.43 \\
(2.79)\end{array}$ \\
\hline \multicolumn{6}{|c|}{ Square Bound by Real Contours } \\
\hline $\begin{array}{c}2.75 \\
(1.54)\end{array}$ & $\begin{array}{c}9.60 \\
(0.74)\end{array}$ & $\begin{array}{c}2.78 \\
(1.63)\end{array}$ & $\begin{array}{c}9.13 \\
(0.78)\end{array}$ & $\begin{array}{c}4.63 \\
(0.54)\end{array}$ & $\begin{array}{c}9.28 \\
(0.91)\end{array}$ \\
\hline
\end{tabular}

*The viewing conditions were arranged such that there was either no disparity cue present or there was a disparity cue that was either compatible or incompatible with the monocular depth cues present in the display.
Table 2

The Effect of Adding Disparity Between the Plane in Which the Black Squares Lie and the Plane of the White Background (and Its Surrounding Frame) on the Mean Judged Clarity of Subjective Contours and on the Judged Depth Relationship*

\begin{tabular}{lllll}
\hline & \multicolumn{2}{c}{$\begin{array}{c}\text { Black Squares } \\
\text { Lie Behind } \\
\text { Black Frame }\end{array}$} & $\begin{array}{c}\text { Black Squares } \\
\text { Project Forward } \\
\text { of Black Frame }\end{array}$ \\
\hline Judged Depth & 3.15 & $(1.46)$ & 1.15 & $(0.99)$ \\
Contour Clarity & 5.00 & $(2.76)$ & 4.85 & $(2.61)$ \\
\hline
\end{tabular}

*Standard deviations are given in parentheses.

influenced the degree of definition or clarity of the subjective contours as measured on the rating scale. For the two levels of disparity employed, there was no significant increase in judged contour clarity over that obtained in the zero-disparity condition $(t=1.31, t=$ 0.77 , both with $19 \mathrm{df}$ ). Even when the stimulus configuration was arranged such that the black squares appeared to float forward of the white background and its surrounding frame (Table 2), there was no significant change in contour clarity compared with the condition in which the white square was located in front of the black squares $(t=0.23, \mathrm{df}=19)$, although there was a clearly perceived change in depth $(t=4.95$, df $=19$, $p<.001)$. This situation is of interest, since the forward-projection of the black squares counteracts to some extent the interposition cue, yet the overall impression of a subjectively bounded white square lying in front of the black squares remains dominant. The interposition cue apparently overrides the disparity cue even under this condition.

Comparison of thestimuli containing incompatible depth cues with the zero-disparity condition (for stimuli yielding subjective contours) indicated no significant change in depth $(\mathrm{t}=1.71, \mathrm{df}=39)$ or in contour clarity $(t=0.98$, df $=39$; Table 1$)$. This supports Coren's hypothesis that there are strong monocular depth cues in the display. These monocular cues become incompatible with the disparity cue when left and right images are interchanged, and they are apparently of sufficient strength to override the tendency for depth reversal to occur.

An additional finding was that the process of outlining the contour of the white square led to a significant increase in perceived depth compared to the corresponding subjective contour condition. This was true for both zero disparity $(t=5.43, \mathrm{df}=19, \mathrm{p}<.001)$ and the two-disparity conditions $(t=5.81, t=6.50$, both with $19 \mathrm{df}$, both with $\mathrm{p}<.001$; Table 1). It appears that Os see less depth at a given level of disparity when the object in depth is defined by subjective contours than when the contours are given in the display.

\section{SUMMARY}

Subjective contours can be perceived stereoscopically. 
Although the presence of the disparity cue alone may be sufficient to generate subjective contours, the addition of stereoscopic cues to figures of the type discussed here does not appear to enhance the clarity of the contour. The results suggest that the tendency to perceive subjective contours reaches a maximum in the monocular viewing condition; adding the disparity cue does not enhance the tendency to perceive a contour above this maximum. The absence of a real contour does, however, appear to attenuate the judged depth of the display as compared to the situation where the contour is clearly marked in. Coren's hypothesis that the presence of monocular depth cues in the display leads to the perception of subjective contours was supported by the data.

\section{REFERENCES}

Coren, S. Subjective contours and apparent depth. Psychological Review, 1972, 79, 359-367.

Gregory, R. L. The intelligent eye. New York: McGraw-Hill, 1970.

Kanizsa, G. Marzini quasi-percettivi in campi con stimolasione omogenea. Rivista di psicologia, 1955, 49, 7-30.

Lawson, R. B., \& Gulick, W. L. Stereopsis and anomalous contour. Vision Research, 1967, 7, 271-297.

Schriever, W. Experimentelle Studien über stereoskopisches Sehen. Zeitschrift für Psychologie, 1925, 96, 113-170.

Shipley, T. Visual contours in homogeneous space. Science, $1965,150,348-350$.

Valyus, N. A. Stereoscopy. London: Focal Press, 1966.

(Received for publication April 24, 1973;

final revision received August 1, 1973.) 